\title{
Molecular Dynamics Study of Line Edge Roughness and the Proximity Effect in Electron Beam Lithography
}

\author{
Sho Hitomi, Katsushi Michishita, Hiroaki Kawata, Yoshihiko Hirai, and Masaaki Yasuda* \\ Department of Physics and Electronics, Osaka Prefecture University \\ 1-1 Gakuen-cho, Naka-ku, Sakai, Osaka 599-8531, JAPAN \\ *yasuda@pe.osakafu-u.ac.jp
}

\begin{abstract}
Pattern formation in electron beam lithography (EBL) is investigated with molecular dynamics simulations. The electron exposure and development process are modeled by polymer chain scission and polymer segment removal from the resist, respectively. The line edge roughness of a sub-10-nm resist pattern is analyzed with the simulations. The proximity effect in EBL is also investigated. The effects of resist molecular size, secondary electron generation, and the substrate material are discussed.
\end{abstract}

Keywords: electron beam lithography, line edge roughness, proximity effect, molecular dynamics, Monte Carlo simulation

\section{Introduction}

Numerical simulations are indispensable for evaluating and predicting the pattern profiles in lithography techniques [1-9]. With the progress of pattern formation techniques, the feature size of the resist pattern has decreased. When the pattern size becomes less than several tens of nano-meters, the behavior of the polymer molecules in the resist becomes crucial to determine the pattern formation process. Conventional simulations that do not introduce the resist molecular structure fail to analyze the pattern profiles.

As a solution to this problem, stochastic simulations include the polymer molecule chain to model the resist structure and the lithography process [10-14]. Stochastic simulations reveal the microscopic material and process effects on pattern formation in photolithography, extreme ultraviolet lithography, and electron beam lithography (EBL). Molecular dynamics (MD) simulations are also a powerful tool to investigate nanometer-scale patterning, and MD simulations have been successfully used to investigate the pattern profiles in nanoimprint lithography [15-17].

In our previous studies, we reported MD simulations of sub-10-nm pattern formation in EBL $[18,19]$. In the simulations, we introduced the effect of electron exposure by chain scission of the polymer molecule and small segments of polymer molecules are removed from the resist structure in the development process.

In the present work, we study in detail the line edge roughness (LER) of a sub-10-nm resist pattern and the proximity effect in EBL with MD simulations. We investigate the effects of resist molecular size, secondary electron generation, and the substrate material.

\section{Simulation Model}

Figure 1 shows the configuration and procedure used for the present simulations. The sample in the simulations is a polymethylmethacrylate (PMMA) resist on a substrate. The film thickness of the PMMA resist is $4 \mathrm{~nm}$. The molecular weight of the initial PMMA polymer is 5000. The 2-nm-wide line pattern is exposed to electrons. The beam edge blur is assumed to be zero.

The simulation model is the same as that described in our previous work [19]. The simulations consist of two parts: electron exposure and resist development. The effect of electron exposure is introduced by chain scission of the PMMA polymer molecules in the MD simulations. In the present simulations, the PMMA film is divided into eight layers. The rate of chain scission in the resist is set to be proportional to the exposure intensity distribution of each layer preliminarily calculated by Monte Carlo simulation of the electron scattering $[1,5]$. 
The breaking positions in the polymer chain are randomly selected. The chain scission and the structural relaxation processes are alternately repeated in the exposure simulation.

The development process is modeled by removing small segments of the polymer molecule from the resist structure. The resist is developed from the surface to the bottom layer. Resist molecules are removed from the surface layer in ascending order of size. First, monomers are removed from the resist, and then dimers, trimers, and tetramers in sequence. The molecule removal and the structural relaxation processes are also alternately repeated in the development simulation. After development of the surface layer finishes, the second layer is developed in the same manner. The same procedures are repeated until the bottom layer is developed. The interaction between the resist and the developer molecules is not included in the present simulations. MD simulation of the development process of PMMA resist have been recently reported [20].

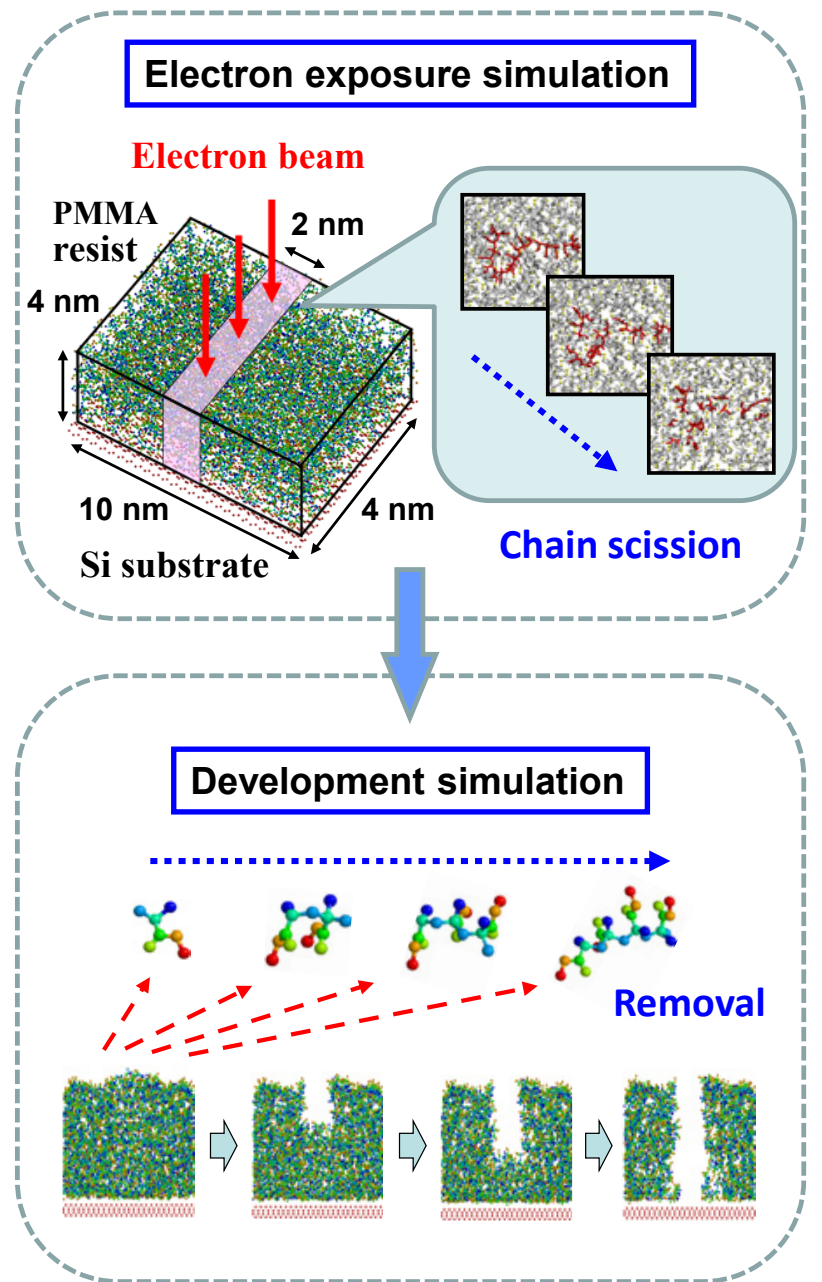

Fig. 1. Configuration and procedure used for the MD simulations. The sample is a PMMA resist on a substrate. The simulation consists of two parts: electron exposure and resist development.
The force field reported by Okada et al. [21] is used for the PMMA resist. It consists of bond stretching, bending, and torsion potentials, and nonbonding interactions including Lennard-Jones and Coulomb potentials. The Lennard-Jones potential is used between the resist polymer and the substrate. The width and depth of the unit structure of the PMMA resist are 10 and $4 \mathrm{~nm}$, respectively. The number of PMMA molecules in the unit structure is 23 . We adopted a periodic boundary condition in the horizontal direction. The temperature is kept constant at $300 \mathrm{~K}$. The time step in the MD simulation is $1 \mathrm{fs}$.

We use two types of Monte Carlo simulations of electron scattering to calculate the exposure intensity distributions. When we do not consider the effect of secondary electron generation, the continuous slowing down approximation model [1] is used. When we introduce the effect of secondary electron generation, the fast secondary electron production model [5] is used.

\section{Results and Discussion}

\section{1. Pattern Edge Structure}

First, we checked the pattern edge structure at the atomic level. Figure 2 shows an enlarged view of the pattern edge structure of the PMMA resist. The change of the same portion of the pattern edge is shown. One molecule is highlighted as a marker molecule. The thermal motion of the marker molecule can be seen. Although the LER at the atomic level is dynamic rather than static, as shown in the figure, the motion is not large at room temperature and the alignment of the molecules in the resist does not change. Only the motions of the side chains of the molecule are significant.

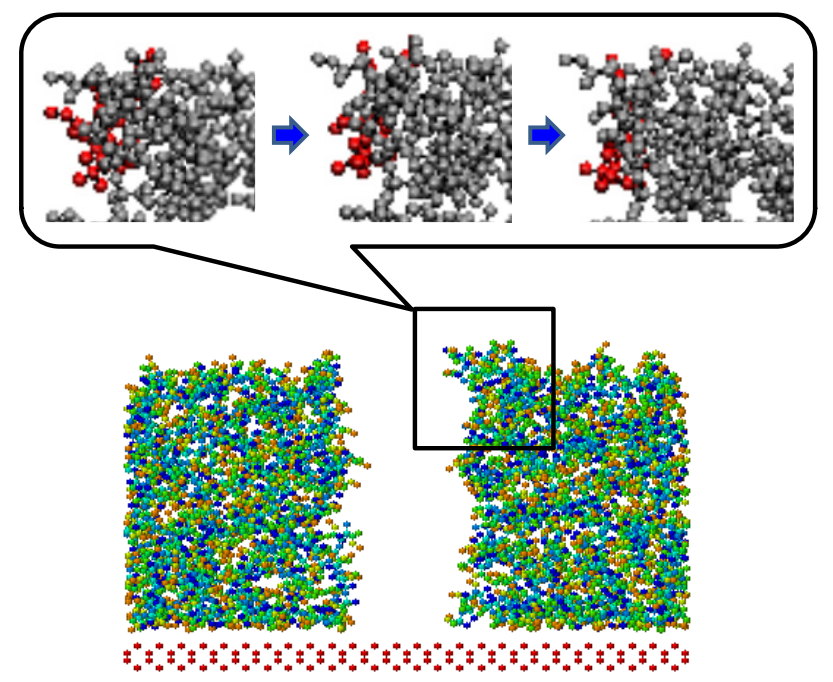

Fig. 2. Transformation of the edge structure of the PMMA resist. One molecule is highlighted as a marker molecule. 


\section{2. Acceleration Voltage Dependence}

Figure 3 shows the LER of the 2-nm-wide line pattern as a function of the acceleration voltage. In this case, we do not include the effect of secondary electron generation in the electron exposure process. Three cases of the maximum polymerization degrees of the removed polymer segment $\left(n_{\max }=4,6\right.$, and 8) are compared. The difference of the maximum polymerization degrees of the removed segment corresponds to the difference of the solubility of the developer or the development time in the actual EBL. The LER increases with increasing acceleration voltage for all of the polymerization degrees. This is because the blur of the pattern edge owing to electron scattering becomes small at high acceleration voltages. The LER values at 10 and $100 \mathrm{kV}$ are almost the same because of the small resist thickness. The LER increases with increasing polymerization degrees of the removed segment at all acceleration voltages. As we will discuss in the next section, the LER at $100 \mathrm{kV}$ is close to the size of the main carbon chain of the removed polymer segment. At high acceleration voltages, the removed polymer size grately affects the LER.

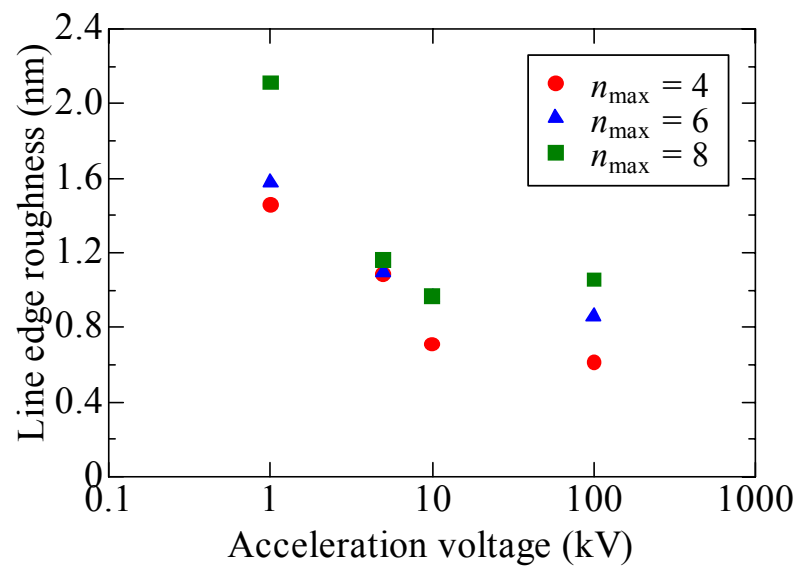

Fig. 3. LER of the 2-nm-wide line pattern as a function of acceleration voltage. Three different maximum polymerization degrees $\left(n_{\max }\right)$ of the removed polymer segment are compared.

\section{3. Resist Molecular Size Effect}

Figure 4 shows the LER of the 2-nm-wide line pattern as a function of polymerization degree of the removed resist molecular segment in the development process. The molecular structures for each polymerization degree are also shown in the figure. The acceleration voltage is $100 \mathrm{kV}$ and the electron dose is $24.7 \mathrm{mC} / \mathrm{cm}^{2}$. Under these exposure conditions, electron scattering and shot noise effects are sufficiently suppressed. The LER linearly increases with increasing polymerization degree of the removed segment. The developed molecular lengths for each polymerization degree are also shown. The molecular length is defined as the main chain length of the molecule for the straight molecule. The developed molecular length also linearly increases with increasing polymerization degree. Although the molecular length is $0.2-0.3 \mathrm{~nm}$ larger than the LER for each polymerization degree, the LER is clearly correlated with the developed molecule length. Thus, when the LER is minimized by optimizing the acceleration voltage and electron dose, the LER is determined by the resist molecule structure.

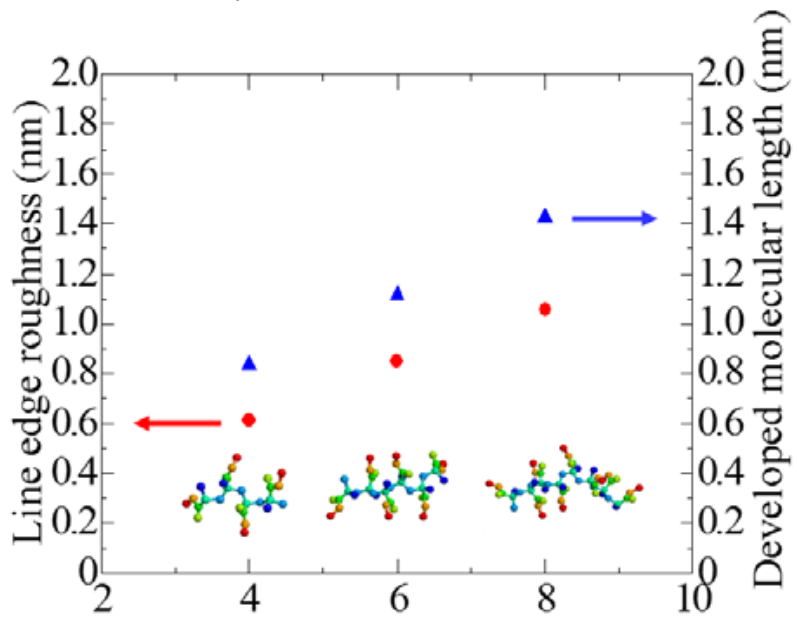

Polymarization degree of developed molecules

Fig. 4. LER of the 2-nm-wide line pattern exposed at $100 \mathrm{kV}$ as a function of polymerization degree of the removed resist molecular segment. The developed molecular lengths for each polymerization degree are also shown.

\section{4. Critical Dimension Error}

We also investigated the critical dimension (CD) error in EBL. Figure 5 shows the CD error as a function of the acceleration voltage for the 2-nm-wide line pattern. The number of chain scissions in the exposed area is about 320 and almost the same for all of the acceleration voltages. The maximum polymerization degree of the developed molecule is 4 . The CD error is defined as the difference between the nominal line edge position and the average position of the edge atoms obtained by the MD simulation. In Figure 5, the CD error decreases with increasing acceleration voltage. When the electron scattering is suppressed at high acceleration voltage, the CD error becomes approximately $0.2 \mathrm{~nm}$, which is less than the developed molecular length in Fig. 4 and close to the length of the side chain of the molecule. 


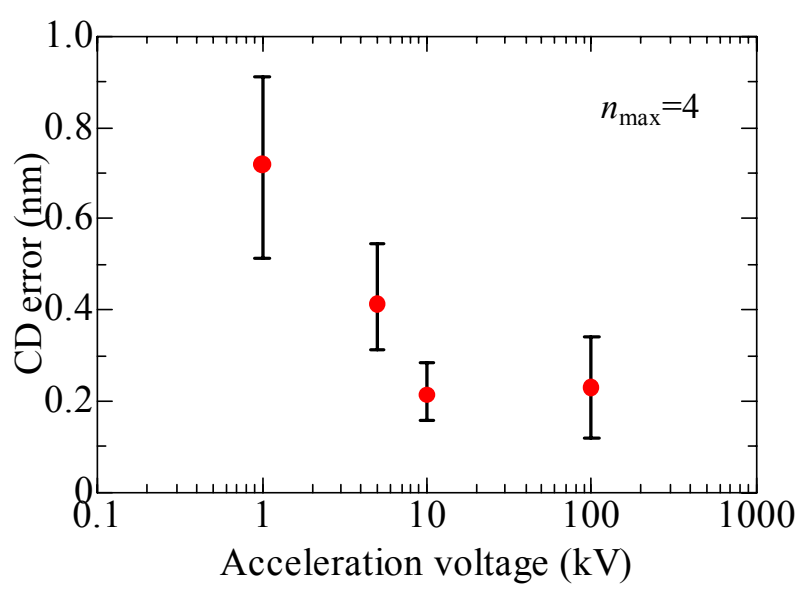

Fig. 5. CD error as a function of acceleration voltage for the 2-nm-wide line pattern.

\section{5. Effect of Secondary Electron Generation}

We then investigated the effect of secondary electron generation on sub-10-nm pattern formation in EBL. Figure 6 shows the exposure intensity distributions in the PMMA resist film on a Si substrate for a 2-nm-wide line pattern at 100 $\mathrm{kV}$ obtained by Monte Carlo simulation of electron scattering. The distributions obtained by the model with and without secondary electron generation are compared. The distribution consists of two parts: a forward scattering part (large narrow distribution) and a backscattering part (small broad distribution). The forward scattering parts are almost the same regardless of whether secondary electron generation is included or not because the PMMA film thickness is quite small. However, the intensity of the backscattering part of the distribution becomes higher when including secondary electron generation.

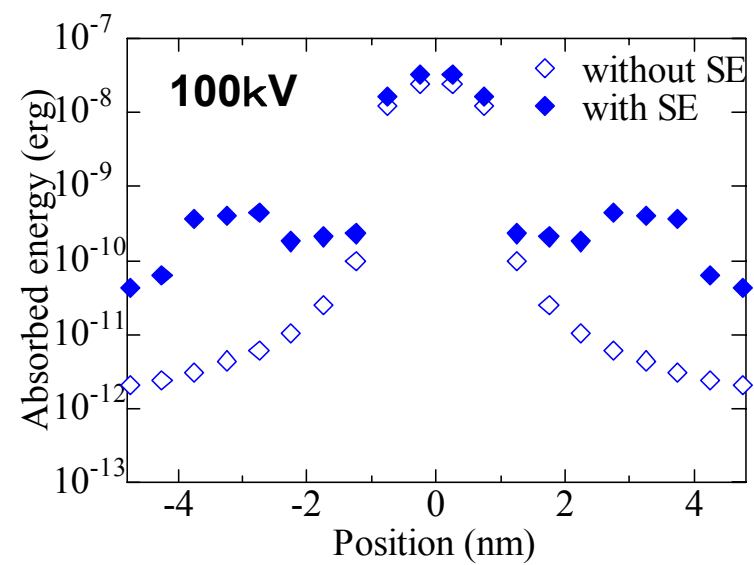

Fig. 6. Exposure intensity distribution of PMMA resist film on Si substrate for a 2-nm-wide line pattern at 100 $\mathrm{kV}$. The distributions with and without secondary electron (SE) generation are compared.
To evaluate the effect of secondary electron generation on LER, we performed MD simulations of pattern formation in EBL based on the above mentioned two exposure intensity distributions. Figure 7 shows top views of 2-nm-wide line patterns in PMMA resists on Si substrates exposed at $100 \mathrm{kV}$. The simulation results with and without secondary electron generation are compared. The maximum polymerization degree of the developed molecule in the development simulation is 4 . The LERs estimated from these pattern structures with and without secondary electron generation are 0.61 and $0.95 \mathrm{~nm}$, respectively. Although secondary electron generation increases the LER in this example, the effect is relatively small because of the thin resist thickness in the simulation.

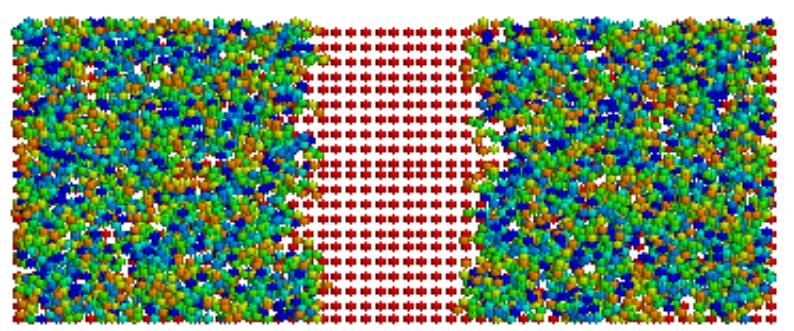

(a)

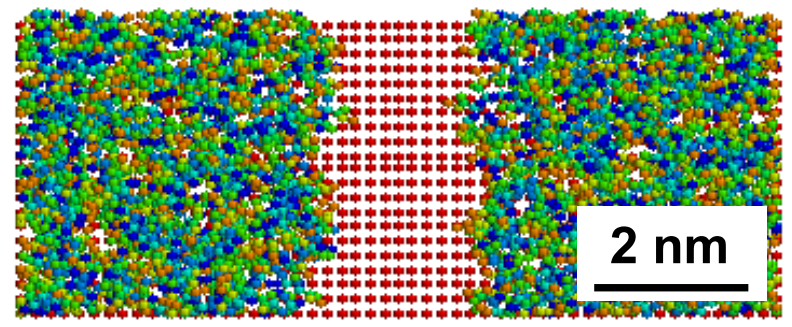

(b)

Fig. 7. Top view of the 2-nm-wide line pattern in the PMMA resist on a Si substrate exposed at $100 \mathrm{kV}$. The simulation results (a) without and (b) with secondary electron generation are compared.

\section{6. Effect of Substrate Material}

The substrate material also affects the LER of resist patterns. The LERs of sub-10-nm patterns on various substrates were investigated. Figure 8 shows the LER of 2-nm-wide line patterns in the PMMA resist on different substrates as a function of acceleration voltage. The free-standing PMMA resist film is compared with PMMA resists on $\mathrm{Si}$ and $\mathrm{Au}$ substrates. Secondary electron generation is not included in the simulation. The maximum polymerization degree of the developed molecule in the development simulation is 4 . When the acceleration voltage is low, the LER of the free-standing PMMA film is the smallest. Because 
of backscattered electrons, the LER of the PMMA resists on the substrates is larger than free-standing resist, but the dependence on the substrate material is small. With increasing acceleration voltage, the LER decreases because forward scattering in the resist becomes small. The effect of the substrate material on LER becomes large at high acceleration voltages. The LER for the $\mathrm{Au}$ substrate becomes larger than that for the $\mathrm{Si}$ substrate at high acceleration voltages.

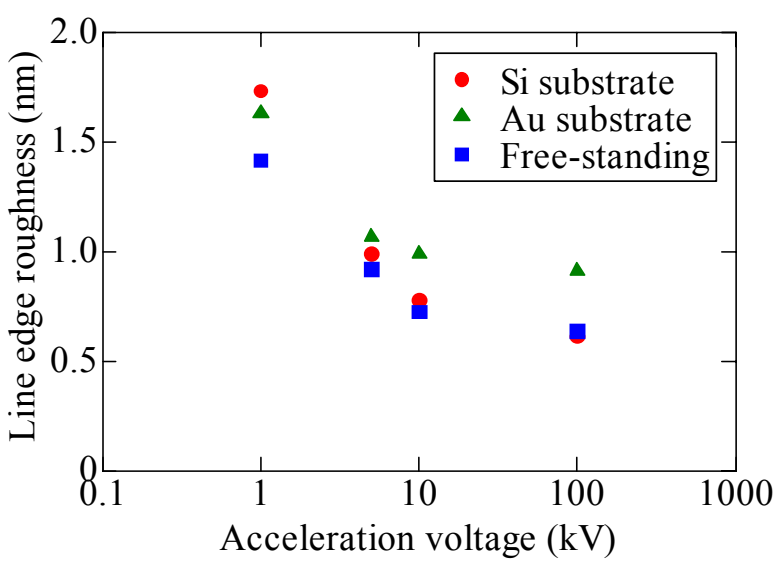

Fig. 8. LER of PMMA resist patterns on various substrates as a function of acceleration voltage.

\section{7. Proximity effect}

Finally, we investigated the proximity effect in sub-10-nm patterning in EBL. Figure 9 shows a schematic diagram of the simulation. Two adjacent 2-nm-wide line patterns in the PMMA resist on the Si substrate are exposed to electrons.

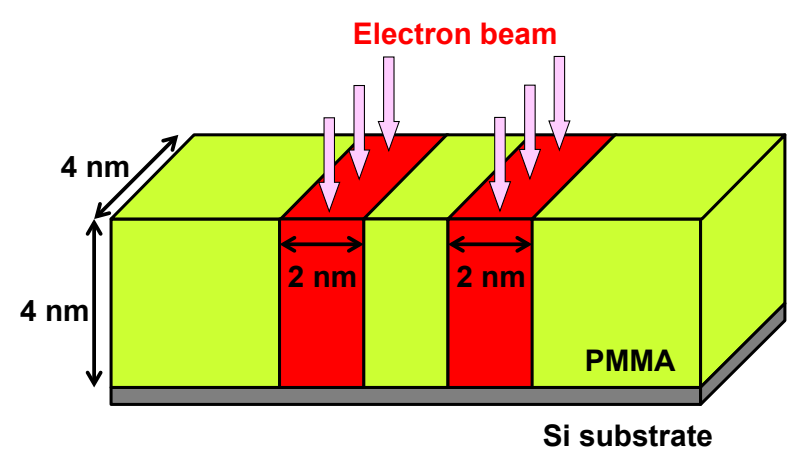

Fig. 9. Schematic diagram of the simulation. Two adjacent 2-nm-wide line patterns in the PMMA resist on a Si substrate are exposed.

Figure 10 shows two adjacent 2-nm-wide line patterns in the PMMA resist on the Si substrate. The intervals between the two lines are 2 and $4 \mathrm{~nm}$. The acceleration voltage of electron exposure is 1 $\mathrm{kV}$. Secondary electron generation is not included. The maximum polymerization degree of the developed molecule in the development simulation is 4 . When the electron dose is not optimized, the resist in the space between the two lines is removed in the case of a 2-nm-wide interval because of the proximity effect, as shown in Fig. 10(a). However, two 2-nm-wide line patterns are formed in the case of a 4-nm-wide interval, as shown in Fig. 10(b).

Figure 11 shows top views of 2-nm-wide line and space patterns in the PMMA resist on the $\mathrm{Si}$ substrate exposed at $5 \mathrm{kV}$. The simulation results with and without secondary electron generation are compared. The maximum polymerization degree of

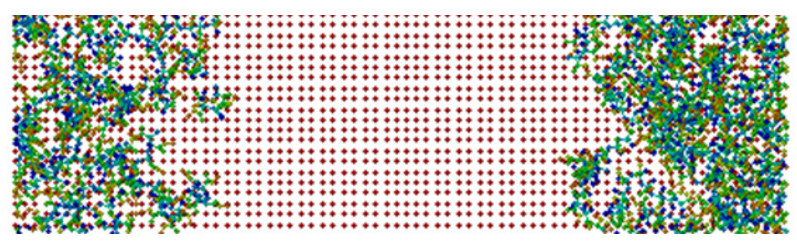

(a)

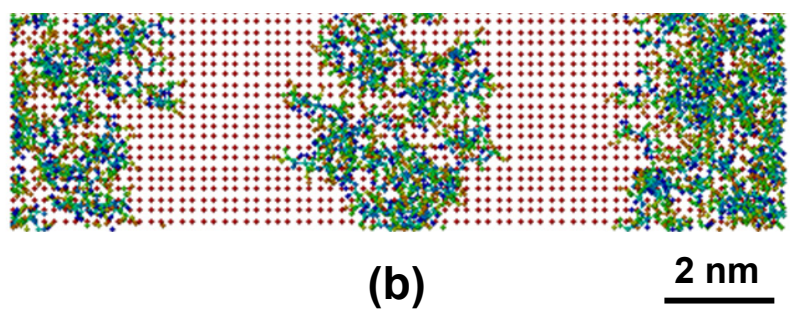

Fig. 10. Top views of two adjacent 2-nm-wide line patterns with intervals of (a) 2 and (b) $4 \mathrm{~nm}$. The acceleration voltage in the electron exposure is $1 \mathrm{kV}$.

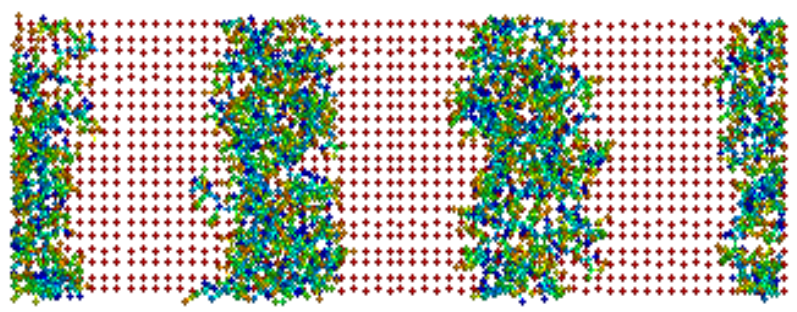

(a)

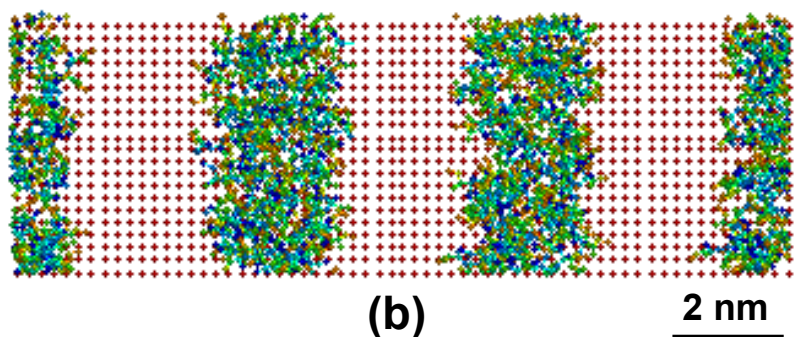

Fig. 11. Top views of 2-nm-wide line and space patterns in the PMMA resist on a $\mathrm{Si}$ substrate exposed at $5 \mathrm{kV}$ : (a) without and (b) with secondary electron generation. 
the developed molecule is 4 . In both cases, 2-nm-wide line and space patterns are formed. As is the case with the LER, the influence of secondary electron generation on the proximity effect is relatively small because of the thin resist thickness in the simulation.

\section{Conclusions}

The LER and proximity effect for sub-10-nm patterning of PMMA resist by EBL was investigated with MD simulations. With increasing acceleration voltage, the LER decreases and becomes close to the developed resist molecular size. When the LER is minimized by optimizing the acceleration voltage and electron dose, there is an obvious correlation between the LER and the resist molecular size. Furthermore, electron backscattering from heavy element substrates increases the LER in sub-10-nm pattern formation by EBL. The influences of secondary electron generation on the LER and the proximity effect are relatively small because of the thin resist thickness in the simulation.

The resist molecular size dependence of the proximity effect is not clarified in the present work. In sub-10-nm lithography, the pattern size becomes comparable to the resist molecular size. It is important to understand the molecular behavior in the resist to determine of the proximity effect correction at the molecular scale.

\section{Acknowledgement}

This work was supported by JSPS KAKENHI Grant number 25249052.

\section{References}

1. K. Murata, E. Nomura, K. Nagami, T. Kato, and H. Nakata, Jpn. J. Appl. Phys., 17 (1978) 1851.

2. E. Nomura, K. Murata, and K. Nagami, Jpn. J. Appl. Phys., 18 (1979) 1353.

3. A. R. Neureuther, D.F. Kyser, and C.H. Ting, IEEE Trans. Electron Devices, ED-26 (1979) 686.
4. W. G. Oldham,
S. N. Nandgaonkar,
A. R.

Neureuther, and M. O'Toole, IEEE Trans. Electron Devices, ED-26 (1979) 717.

5. K. Murata, D. F. Kyser, and C. H. Ting, J. Appl. Phys., 52 (1981) 4396.

6. G. Owen and P. Rissman, J. Appl. Phys., 54 (1983) 3573.

7. M. Kotera, T. Yamada, and Y. Ishida, Jpn. J. Appl. Phys., 41 (2002) 4150.

8. M. Kotera, K. Yagura, H. Ttanaka, D. Kawano and T. Maekawa, Jpn. J. Appl. Phys., 47 (2008) 4944.

9. M. Stepanova, T. Fito, Zs. Szabó, K. Alti, A. P. Adeyenuwo, K. Koshelev, M. Aktary and S. K. Dew, J. Vac. Sci. Technol., B 28 (2010) C6C48.

10. G. Wallraff, J. Hutchinson, W. Hinsberg, F. Houle, P. Seidel, R. Johnson, W. Oldham, J. Vac. Sci. Technol., B 12 (1994) 3857.

11. G. P. Patsis, Polymer, 46 (2005) 2404.

12. G. P. Patsis and E. Gogolides, J. Vac. Sci. Technol., B 23 (2005) 1371.

13. N. Tsikrikas, D. Drygiannakis, G. P. Patsis, I. Raptis, S. Stavroulakis, and E. Voyiatzis, Jpn. J. Appl. Phys., 46 (2007) 6191.

14. G.P. Patsis, D. Drygiannakis, I. Raptis, Microelectron. Eng., 87 (2010) 989.

15. J. -H. Kang, K. -S. Kim and K. -W. Kim, Tribol. Lett., 25 (2007) 93.

16. A. Taga, M. Yasuda, H. Kawata and Y. Hirai, $J$. Vac. Sci. Technol., B 28 (2010) C6M68.

17. M. Yasuda, K. Araki, A. Taga, A. Horiba, H. Kawata and Y. Hirai, Microelectron. Eng., 88 (2011) 2188.

18. M. Yasuda, H. Sakai, R. Takai, H. Kawata and Y. Hirai, Microelectron. Eng., 112 (2013) 287.

19. K. Michishita, M. Yasuda, H. Kawata and Y. Hirai, Jpn. J. Appl. Phys., 53 (2014) 06JB02.

20. M. A. Mohammad, K. P. Santo, S. K. Dew, and M. Stepanova, J. Vac. Sci. Technol., B 30 (2012) 06FF11.

21. O. Okada, K. Oka, S. Kuwajima, S. Toyoda and K. Tanabe, Comput. Theo. Polymer Sci., 10 (2000) 371. 